\title{
Wasserqualität und Wasseraufbereitung
}

Ein in den vergangenen Jahren immer öfter eingesetztes Verfahren zur Aufbereitung von Wasser und Abwasser ist die Membrantechnik. Die nach Bedarf auswählbare Trenngrenze einerseits sowie die Möglichkeit, die Membrantechnik mit anderen Verfahren zu kombinieren, schafft den Vorteil eines breit gestreuten Einsatzbereiches. Aufgrund weltweiter Prognosen ist zu erwarten, dass ein verstärktes Heranziehen der Membrantechnik zur Wasser- und Abwasseraufbereitung zu sinkenden Kosten für den Ver- und Entsorgungsbereich führen wird.

Die Nutzung von Quell- und Grundwasser aus Kluftgrundwasserleitern und Karstgebieten ist für die kommunale Wasserversorgung in Süddeutschland charakteristisch. Aufgrund der unzureichenden Überdeckung mit filtrierenden Bodenschichten kommt es in diesen Gebieten nach starken Regenfällen und bei der Schneeschmelze oft mehrmals zu Eintrübungen der Rohwässer. Die Beherrschung dieses Phänomens mittels Flockungsfiltration erfordert eine kontinuierliche Beobachtung des Rohwassers und eine auf die jeweilige Beschaffenheit abgestimmte Anpassung des Betriebes. Kleine Wasserversorgungsunternehmen können dies nicht mit der erforderlichen Sicherheit gewährleisten, sodass nach Alternativen gesucht werden muss. Umfangreiche, rund zehnjährige Erfahrungen mit dem Einsatz von Ultrafiltrationsanlagen in Bayern veranschaulichen, dass mithilfe dieser Art der Wasseraufbereitung eine gesicherte Entfernung der Partikel aus den unterschiedlichsten Rohwässern möglich ist (Beitrag Krause). Dadurch wird gewährleistet, dass die nachfolgende Desinfektion den geforderten Wirkungsgrad erreicht.

Hand in Hand mit der Forderung nach einer flächendeckenden Abwasserentsorgung ergibt sich die Notwendigkeit, verstärkter als bisher, Augenmerk auf den mikrobiologisch-hygienischen Gütezustand des gereinigten Abwassers zu lenken. Der Grund dafür liegt u. a. darin, dass die besagte Forderung auch für dünn besiedelte Gebiete und Extremlagen gilt, wo eine Versickerung des gereinigten Abwassers oft als einzig mögliche Maßnahme angesehen wird. Da konventionelle Abwasserreinigungsverfahren jedoch keine ausreichend gesicherte Entfernung von hygienisch relevanten Mikroorganismen bieten, muss nach Alternativen gesucht werden. Als solche bieten sich Membranbelebungsanlagen an. Trotz mehrfach beschriebener guter mikrobiologischer Reinigungsleistung dieses Verfahrens besteht noch eine Reihe von offenen Fragen, die sich im Wesentlichen auf das Ausmaß und die Beständigkeit der Reinigungsleistung beziehen. Im Rahmen eines vom Bundesministerium für Land- und Forstwirtschaft, Umwelt und Wasserwirtschaft geförderten Projektes sollte geklärt werden, welche Eliminationsraten für hygienisch relevante Mikroorganismen bei unterschiedlichen Betriebsbedingungen erreicht werden und welche Bedeutung den jeweiligen Ablaufwerten beizumessen ist. Der Artikel von Pressl et al. enthält die im Zuge des genannten Projektes gewonnenen Erkenntnisse zum Anlagenbetrieb von Membranbioreaktoren bei kleinen Anschlussgrößen.

Mikrobiologische Verunreinigungen von Wasser stellen nach wie vor ein wesentlich höheres direktes Gesundheitsrisiko für die Bevölkerung dar, als jeder andere unerwünschte Inhaltsstoff. Neue Formen von Erregern und damit verbunden neue Risiken für Erkrankungen einerseits, sowie erhöhte Anforderungen an die Beurteilung des Gütezustandes von Wasser machen es erforderlich, sich verstärkt mit dem mikrobiologischen Gütezustand von Wasser auseinander zu setzen. Für den Nachweis von Mikroorganismen werden entweder kulturabhängige oder nicht kulturabhängige Verfahren herangezogen. Bei der klassischen bakteriologischen Standardanalytik wird der Gehalt an Mikroorganismen in einer Wasserprobe nach Anzucht auf allgemeinen oder selektiven Nährmedien qualifiziert und quantifiziert. Eine einfache Möglichkeit die Aussagekraft eines mikrobiologischen Standardparameters zu 
erhöhen besteht darin, die vorgegebene Volumeneinheit zu erhöhen (Beitrag Zibuschka/Lindner). Bewährt hat sich diese Vorgehensweise dort, wo aufgrund der Befundlage geklärt werden soll, ob eine auf niedrigem Niveau liegende, permanente fäkale Belastung vorliegt oder ob es sich um eine zeitlich begrenzte Beeinflussung des Wassers mit einer entsprechend zeitlich begrenzten Ursache handelt.

Sogenannte alternative Methoden bedienen sich anderer Nachweisprinzipien wie z. B. dem Nachweis der metabolischen Aktivität der Enzyme B-D-Galactosidase und B-D-Glucuronidase zur Bestimmung von E. coli und coliformen Bakterien. Die Arbeit von Zibuschka et al. berichtet über Untersuchungsergebnisse, die mittels eines Messgerätes (COLIGUARD ${ }^{\circledR}$ EC.s, mbOnline GmbH, St. Pölten) für ein Near-Real-Time-Monitoring (NRTM) erhalten wurden. Als Untersuchungsobjekt diente ein für Erholungszwecke genutztes Oberflächengewässer. Die im Zeitraum von August bis November 2009 durchgeführten Untersuchungen zeigen eine gute Übereinstimmung zwischen Ergebnissen, die mittels NRTM und parallel durchgeführten konventionellen E.-coli-Bestimmungen erhalten wurden.

\section{Dr. Franziska Zibuschka}

Universität für Bodenkultur Wien

Department Wasser-Atmosphäre-Umwelt

Institut für Siedlungswasserbau, Industriewasserwirtschaft und Gewässerschutz

Muthgasse 18

1190 Wien

franziska.zibuschka@boku.ac.at 